\title{
Hydrogen motions in the $\alpha$-relaxation regime of poly(vinyl ethylene): A molecular dynamics simulation and neutron scattering study
}

\author{
A. Narros \\ Departamento de Física de Materiales UPV/EHU, Apartado 1072, 20080 San Sebastián, Spain \\ F. Alvarez \\ Departamento de Física de Materiales UPV/EHU, Apartado 1072, 20080 San Sebastián, Spain \\ and Unidad Física de Materiales (CSIC-UPV/EHU), Apartado 1072, 20080 San Sebastián, Spain \\ A. Arbe \\ Unidad Física de Materiales (CSIC-UPV/EHU), Apartado 1072, 20080 San Sebastián, Spain \\ J. Colmenero \\ Departamento de Física de Materiales UPV/EHU, Apartado 1072, 20080 San Sebastián, Spain; \\ Unidad Física de Materiales (CSIC-UPV/EHU), Apartado 1072, 20080 San Sebastián, Spain; \\ and Donostia International Physics Center, Apartado 1072, 20080 San Sebastián, Spain \\ D. Richter \\ Institut für Festkörperforschung, Forschungszentrum Jülich GmbH, D-52425 Jülich, Germany \\ B. Farago \\ Institut Laue-Langevin, Boîte Postale 156, 38042 Grenoble Cedex 9, France
}

(Received 3 March 2004; accepted 20 May 2004)

\begin{abstract}
The hydrogen motion in poly(vinyl ethylene) (1,2-polybutadiene) in the $\alpha$-relaxation regime has been studied by combining neutron spin echo (NSE) measurements on a fully protonated sample and fully atomistic molecular dynamics simulations. The almost perfect agreement between experiment and simulation results validates the simulated cell. A crossover from Gaussian to non-Gaussian behavior is observed for the intermediate scattering function obtained from both NSE measurements and simulations. This crossover takes place at unusually low $Q$ values, well below the first maximum of the static structure factor. Such anomalous deviation from Gaussian behavior can be explained by the intrinsic dynamic heterogeneity arising from the differences in the dynamics of the different protons in this system. Side group hydrogens show a markedly higher mobility than main chain protons. Taking advantage of the simulations we have investigated the dynamic features of all different types of hydrogens in the sample. Considering each kind of proton in an isolated way, deviations from Gaussian behavior are also found. These can be rationalized in the framework of a simple picture based on the existence of a distribution of discrete jumps underlying the atomic motions in the $\alpha$ process. (c) 2004 American Institute of Physics. [DOI: 10.1063/1.1772761]
\end{abstract}

\section{INTRODUCTION}

The freezing of the structural $(\alpha)$ relaxation in a glassforming system leads to the glass transition phenomenonone of the most intriguing problems in condensed matter physics. Therefore, the understanding of the molecular motions during this relaxation is of utmost importance to shed some light on the glass formation process. This can be facilitated by using microscopic techniques such as neutron scattering (NS), which provides spatial information at atomic level, and molecular dynamics (MD) simulations. Though both techniques have proven to be extremely useful for studying the dynamics of glass forming systems in the $\alpha$ relaxation, the deepest insight into this problem is naturally achieved by the combination of the two approaches. In this work we show the potential of such a combination for unraveling the origin of the deviations from Gaussian behavior found for the hydrogens of poly(vinyl ethylene) (or 1,2polybutadiene) (PVE).

The knowledge on the self-motion of hydrogens in glass forming polymers acquired during last years allows to qualify these motions in the $\alpha$-relaxation regime as anomalous diffusion. ${ }^{1,2}$ This is based on NS results on a variety of polymers and, also recently, on MD simulations. ${ }^{3}$ The stretching of the self-correlation function $F_{s}(Q, t)$ relating to the moving hydrogens is well described in terms of a Kohlrausch-Williams-Watts (KWW) function,

$$
F_{s}(Q, t)=A(Q) \exp \left(-\left[\frac{t}{\tau_{w}(Q)}\right]^{\beta}\right)
$$

Here $\beta$ is the stretching parameter $(0<\beta \leqslant 1), \tau_{w}(Q)$ the relaxation time, and

$$
A(Q)=\exp \left(-\frac{\left\langle u^{2}\right\rangle}{3} Q^{2}\right)
$$

an effective Debye-Waller factor (DWF) describing the initial decay of $F_{s}(Q, t)$ due to fast librational and vibrational 


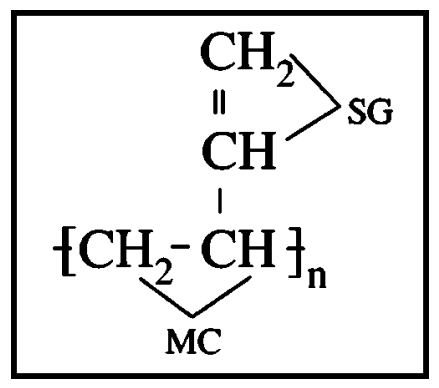

FIG. 1. Chemical composition of PVE. The locations of the hydrogens designed as "main chain" (MC) and "side group" (SG) are shown.

motions of the protons. $\left\langle u^{2}\right\rangle$ is the associated mean squared displacement. For a large number of polymers it was established that $\tau_{w}(Q)$ follows a power law

$$
\tau_{w} \propto Q^{-2 / \beta}
$$

for not too large $Q$ values $\left(Q \leqslant 1.3, \ldots, 1.5 \AA^{-1}\right)$. This dispersion, together with the stretched character of the scattering function, implies Gaussian behavior of the corresponding van Hove correlation function. These observations suggest a scenario of sublinear diffusion for atomic motions in the $\alpha$-relaxation regime, where the mean squared displacement increases sublinearly in time according to $\left\langle r^{2}(t)\right\rangle \propto t^{\beta}{ }^{1,2}$

Recently, in a combined study of the self-motion of main chain protons in polyisoprene (PI) by NS and MD simulations, deviations from this $Q$ dependence of $\tau_{w}$ were found for $Q \geqslant 1.3 \AA^{-1}$. 3,4 These deviations from Gaussian behavior could be interpreted as due to the existence of a distribution of finite jump lengths $\ell$ underlying the sublinear diffusion of the proton motion. In the frame of such a simple model the main features of the non-Gaussian parameter calculated from the simulations could be reproduced. It is noteworthy that similar features have also been reported for different systems such as Lennard-Jones liquids, ${ }^{5}$ water, ${ }^{6}$ ortho-terphenyl, ${ }^{7}$ and selenium. ${ }^{8}$

An interesting question now is how these deviations from Gaussian behavior manifest in a polymer containing protons with markedly different mobilities, as it is the case for main chain and side group protons. In such a case, stronger deviations from Gaussian behavior are expected which should arise from the heterogeneity in the dynamics originating from the chemical structure of the polymer. With these ideas in mind, we have performed a careful study on the proton dynamics of PVE (see chemical structure in Fig. 1) well above the glass transition temperature $T_{g}\left(T_{g}\right.$ $=272 \mathrm{~K}$ ), by combining NS measurements and MD simulations. We emphasize that the desired information is only available if the MD simulations are fully atomistic. We follow the strategy to first validate the MD simulations by direct comparison with experimental results in the dynamic range of interest. Once the reliability of the simulated cell is proven, we extract information from the simulated atomic trajectories that cannot be accessed experimentally.

The paper is organized as follows: In the following section the simulation details and the definition of some of the functions appearing later are given. Thereafter a section is devoted to the experimental aspects concerning the sample and the neutron spin echo (NSE) technique. The results obtained from experiments and simulations are compared in the following section, leading to the validation of the latter. Taking advantage of the validation of the simulation, we have used it to go beyond the experimental capabilities. In the discussion section the sources of non-Gaussian behavior observed are elucidated, and the different dynamics of the different kind of hydrogens in the sample is addressed.

\section{MOLECULAR DYNAMICS SIMULATIONS}

\section{A. Simulation method}

The simulations were carried out by using the INSIGHT (INSIGHT II 4.0.0 P version) and the DISCOVER-3 module from Molecular Simulations Inc. (now Accelrys) with the Polymer Consortium Force Field (PCFF). Most parameters of this field were derived based on ab initio data using a leastsquares-fit technique developed by Hagler and co-workers. The functional form includes terms which can be divided into two categories-valence terms including diagonal and off-diagonal cross-coupling terms and nonbonded interactions terms. The valence terms represent internal coordinates of bond, angle, torsion angle, and out of plane angle, and the cross-coupling terms include combinations of two or three internal coordinates. The cross-coupling terms are important for predicting vibration frequencies and structural variations associated with conformational changes. The analytical expression employs quartic polynomials for bond stretching and angle bending and a three-term Fourier expansion for torsions. The nonbonded interaction terms include a Coulombic function for the electrostatic interaction and a LennardJones 6-9 function rather than the more customary LennardJones 6-12, for the van der Waals term. More information about this kind of force fields, including the complete analytical expression for the functional form, can be found in Refs. 9 and 10. The model system was built by means of the well known amorphous cell protocol, which was proposed for the first time by Theodorou and Suter. ${ }^{11}$ In this work, we constructed a cubic cell containing one polymer chain of 130 monomer units $\left[-\mathrm{CH}_{2}-\mathrm{CH}\left(\mathrm{CH}=\mathrm{CH}_{2}\right)-\right]_{130}$ at $418 \mathrm{~K}$ and with periodic boundary conditions. The density was ( $\rho$ $=0.813 \mathrm{~g} / \mathrm{cm}^{3}$ ), as extrapolated to $418 \mathrm{~K}$ from the available experimental data. ${ }^{12}$ Such a density leads to a cell dimension of $24.306 \AA$ of side. Standard minimization procedures (Polak-Ribiere conjugate gradients method) were followed in order to minimize the so obtained energy structure, and subsequent dynamics was run for $1 \mathrm{~ns}$ in order to equilibrate the sample. The chosen temperature is high enough to allow local structural equilibration of the sample in this time. ${ }^{13}$ The system obtained in this way was used as a starting point for collecting data every 0.01 ps during a MD run of $1 \mathrm{~ns}$. As integration method we have used the velocity-Verlet algorithm with a time step of $1 \mathrm{fs}$. The simulations were carried out in the NVT ensemble. However, instead of a real temperature-bath coupling (Nose-Hoover or Berendsen thermostats, for instance) in order to control the temperature, we have followed a rather crude velocity scaling procedure but with a wide temperature window of $10 \mathrm{~K}$. Under these conditions, greater temperature fluctuations are allowed but the 
trajectory is disturbed less. In fact, we have checked that by following this simple procedure we obtain results similar to those obtained with a NVE ensemble, which has the proper Newtonian dynamics (this was extensively shown for PI in Ref. 3). After the first $1 \mathrm{~ns}$ MD run, a successive run of $2 \mathrm{~ns}$ was carried out, collecting data every 0.05 ps. In addition, a different cell at $368 \mathrm{~K}\left(\rho=0.844 \mathrm{~g} / \mathrm{cm}^{3}\right.$ and cell dimension of $24.004 \AA$ of side) was constructed by the same protocol and equilibration procedure but starting from the last conformation after the MD of $2 \mathrm{~ns}$ run at $418 \mathrm{~K}$ of the parent chain. A similar procedure was followed at $340 \mathrm{~K}(\rho$ $=0.862 \mathrm{~g} / \mathrm{cm}^{3}$ and cell dimension of $23.836 \AA$ of side) and $320 \mathrm{~K}\left(\rho=0.875 \mathrm{~g} / \mathrm{cm}^{3}\right.$ and cell dimension of $23.718 \AA$ of side).

\section{B. Functions: Dynamic magnitudes from the atomic trajectories}

The trajectory in space of a single atom is described by the self part of the van Hove correlation function $G_{s}(\vec{r}, t)$. $G_{s}(\vec{r}, t)$ is the probability to find an atom at time $t$ at a position $\vec{r}$ if it was at $\vec{r}=0$ for $t=0$ :

$$
G_{s}(\vec{r}, t)=\frac{1}{N}\left\langle\sum_{i=1}^{N} \delta\left[\vec{r}-\left(\vec{r}_{i}(t)-\vec{r}_{i}(0)\right)\right]\right\rangle .
$$

Here $\vec{r}_{i}$ is the position of the atom $i$ and $N$ the number of nuclei in the sample. The brackets denote the ensemble average. $G_{s}(\vec{r}, t)$ can easily be calculated from the MDsimulation trajectories, but, with the exception of some special cases like, for instance, colloids with video microscopy ${ }^{14,15}$ it cannot be accessed experimentally. The accessible information from neutron scattering techniques is provided by the Fourier transforms of $G_{s}(\vec{r}, t)$ in $\vec{Q}$ space: in the time domain, the intermediate scattering function

$$
F_{s}(\vec{Q}, t)=\int G_{s}(\vec{r}, t) \exp (i \vec{Q} \vec{r}) d \vec{r}
$$

can be measured by NSE techniques; on the other hand, time of flight (TOF) or backscattering (BS) techniques asses its counterpart in the frequency domain, the incoherent scattering function

$$
S_{s}(\vec{Q}, \omega)=\frac{1}{2 \pi \hbar} \int G_{s}(\vec{r}, t) \exp [i(\vec{Q} \vec{r}-\omega t)] d \vec{r} d t .
$$

Here, $\hbar \vec{Q}$ and $\hbar \omega$ are the momentum transfer and energy transfer in the scattering experiment, respectively (see Refs. 16-18). As we will compare MD simulation with experimental NS results, we will introduce in this section also some magnitudes and functions related with NS that will have to be invoked in the following. The experimentally accessed magnitude by NS is the double differential scattering cross section. In the general case, it contains an incoherent contribution reflecting the self-motion of atoms, as above defined, and a coherent contribution related to the atomic pair correlations. These contributions are weighted by the factors $I_{i n c}$ and $I_{c o h}(Q)$, respectively. These factors depend on the incoherent and coherent scattering lengths $b_{i}^{i n c}$ and $b_{i}^{\text {coh }}$ of the nuclei in the sample ( $i$ refers to the nucleus considered), and, in addition, $I_{c o h}(Q)$ reveals directly the static structure factor $S(Q)$. They read

$$
I_{\text {inc }}=\frac{1}{N} \sum_{i}^{N}\left(b_{i}^{i n c}\right)^{2}
$$

and

$$
I_{c o h}(Q)=\frac{1}{N}\left\langle\sum_{i}^{N} \sum_{j}^{N} b_{i}^{c o h} b_{j}^{c o h} e^{i \vec{Q}\left(\vec{r}_{i}-\vec{r}_{j}\right)}\right\rangle \propto S(Q) .
$$

Here, $\vec{r}_{i}$ and $\vec{r}_{j}$ are taken at the same time and the brackets mean the thermal average.

For some simple cases-free nuclei in a gas, harmonic crystals, simple diffusion at long times- $G_{s}(\vec{r}, t)$ is a Gaussian function; ${ }^{17,19}$ in an isotropic system this implies

$$
G_{s}^{\text {Gauss }}(r, t)=\left[\frac{\alpha(t)}{\pi}\right]^{3 / 2} \exp \left[-\alpha(t) r^{2}\right] .
$$

In the Gaussian approximation the even moments of $G_{s}(r, t)$,

$$
\left\langle r^{2 n}\right\rangle=\int_{0}^{\infty} r^{2 n} G_{s}(r, t) 4 \pi r^{2} d r
$$

can be straightforwardly calculated. For instance, the mean squared displacement of the atom $\left\langle r^{2}(t)\right\rangle$ is given by $\left\langle r^{2}(t)\right\rangle=3 /[2 \alpha(t)]$. Moreover, in such Gaussian case $F_{s}(Q, t)$ is entirely determined by $\left\langle r^{2}(t)\right\rangle$,

$$
F_{s}^{\text {Gauss }}(Q, t)=\exp \left[-\frac{\left\langle r^{2}(t)\right\rangle}{6} Q^{2}\right] .
$$

In more general cases, deviations of $G_{s}(r, t)$ from the Gaussian form [Eq. (9)] may be expected. $F_{s}(Q, t)$ can then be expressed in terms of its expansion in $Q$ (see, e.g., Ref. 20),

$$
F_{s}(Q, t)=\exp \left[-\frac{\left\langle r^{2}(t)\right\rangle}{6} Q^{2}+\frac{\alpha_{2}(t)\left\langle r^{2}(t)\right\rangle^{2}}{72} Q^{4}+\cdots\right],
$$

where $\alpha_{2}(t)$ giving the leading correction is the so called second order non-Gaussian parameter. It is defined as ${ }^{21}$

$$
\alpha_{2}(t)=\frac{3}{5} \frac{\left\langle r^{4}(t)\right\rangle}{\left\langle r^{2}(t)\right\rangle^{2}}-1 .
$$

Evidently, in the Gaussian case $\alpha_{2}(t)=0$. We note that the calculation of the non-Gaussian parameter can be performed in a straightforward way when the atomic coordinates in real space are known, as it is the case of MD simulations. However, it is extremely difficult to deduce from experimental results since they access the scattering functions in $Q$ space and in a restricted $Q$ range. ${ }^{3,20}$

\section{EXPERIMENT}

\section{A. Sample}

The poly(vinyl ethylene) sample was prepared following well established high-vacuum procedures ${ }^{22}$ by anionic polymerization. Details of synthesis and characterization are given elsewhere. ${ }^{23}$ Purified sec-butyllithium was used as ini- 
tiator. A cyclohexane/benzene mixture served as polymerization solvent. Diethyl ether, tetrahydrofuran, and dipiperidinoethane were used as polar modifiers. The number average molecular weight $M_{n}$ was measured by membrane osmometry in toluene at $37^{\circ} \mathrm{C}$ yielding $M_{n}=20000 \mathrm{~g} / \mathrm{mol}$. The molecular weight distribution was analyzed by size exclusion chromatography. The polydispersity index found was smaller than 1.05 , indicating a near monodisperse distribution. By ${ }^{13} \mathrm{C}$ - and ${ }^{1} \mathrm{H}$-NMR spectroscopy the microstructural composition was determined to correspond to a $95 \%$ content of 1,2-vinyl units. Finally, the glass transition temperature $T_{g}$ was found $T_{g}=272 \mathrm{~K}$ by means of DSC.

\section{B. Neutron spin echo}

Neutron spin echo is distinguished from all other dynamic neutron scattering techniques in that it measures directly the velocity change of each neutron individually during scattering. ${ }^{24-26}$ This allows a decoupling of the neutron bandwidth utilized by the instrument from the obtainable resolution. The velocity measurements are performed using the Larmor precession angle of the neutron spin in a magnetic field. A comparison of these Larmor angles before and after scattering leads to a direct measurement of velocity differences, irrespective of the initial velocity of neutrons.

Thus, a singular feature of NSE is that it gives information in Fourier space and measures the time-dependent correlation functions. Ideally, the NSE signal is ${ }^{24}$

$$
S_{N S E}(Q, t)=\frac{I_{c o h}(Q) \tilde{F}(Q, t)-\frac{1}{3} I_{i n c} F_{s}(Q, t)}{I_{c o h}(Q)-\frac{1}{3} I_{i n c}},
$$

where $\widetilde{F}(Q, t)$ and $F_{s}(Q, t)$ are the intermediate pair correlation function (normalized to its value at $t=0$ ) and the selfcorrelation function above defined, respectively. $I_{c o h}(Q)$ and $I_{\text {inc }}$ denote again the coherent and incoherent intensities. The time variable depends on the precession field $B$, the wavelength $\lambda$, and the length of the precession field $L$ : $t \sim B L \lambda^{3}$. We note that, in practice, the normalization experimentally performed may not be completely correct. As a result of the polarization analysis after the scattering process, a NSE spectrometer offers only a limited bandpass to the scattered neutrons. Inelastically scattered neutrons with wavelengths $\lambda$ shorter than those that the bandpass allows $\lambda_{B P}$ are not transmitted through the instrument and do not participate in the normalization event.

Hydrogen presents a very large value for the incoherent scattering length $\left(b_{H}^{i n c}=25.274 \mathrm{fm}\right)$. In a fully protonated polymer sample consisting of $\mathrm{H}$ and $\mathrm{C}$, the scattering is completely dominated by the incoherent scattering from the protons (C only contributes coherently with $b_{C}^{\text {coh }}=6.6511 \mathrm{fm}$ and $\left.b_{H}^{c o h}=-3.7406 \mathrm{fm}\right)$. Therefore, in such case $I_{i n c}$ $\gg I_{c o h}(Q)$ for all $Q$ values and the NSE signal directly measures $F_{s}(Q, t)$ related to the self-motion of the hydrogens (except for corrections for the amplitude).

The measurements were carried out by means of the NSE instrument IN11C at the ILL in Grenoble (France). ${ }^{25}$ The multidetector at IN11C covers an angular range of $30^{\circ}$ in the horizontal plane. The selector provides a monochromatization of $\Delta \lambda / \lambda=15 \%$. With an incident wavelength $\lambda$

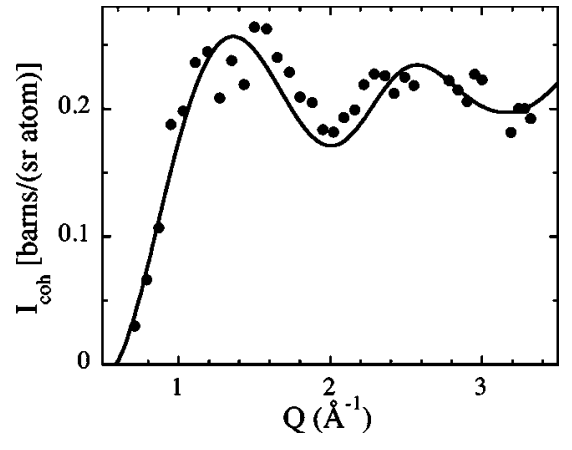

FIG. 2. Coherent scattering intensity measured by DNS on the protonated PVE sample (dots) and calculated from the MD simulations (solid line).

$=5.6 \AA$, the multidetector was placed at $32^{\circ}, 60^{\circ}$, and $90^{\circ}$ scattering angle $\theta$ for its central detector. Thereby, a $Q$ range $[Q=4 \pi \sin (\theta / 2) / \lambda] \quad 0.33 \leqslant Q \leqslant 1.78 \AA^{-1}$ and a time range $5.4 \mathrm{ps} \leqslant t \leqslant 1.5 \mathrm{~ns}$ were explored. Smaller $Q$ values were accessed by using another incident wavelength, $\lambda=8 \AA$, with the center of the multidetector at $\theta=32^{\circ}$. The range 0.23 $\leqslant Q \leqslant 0.63 \AA^{-1}$ for times $14 \mathrm{ps} \leqslant t \leqslant 2.7 \mathrm{~ns}$ was covered in this way. Such measurements were performed at $368 \mathrm{~K}$ and $418 \mathrm{~K}$. At lower temperatures the slowing down of the relaxation prevented the resolution of the decays in the low $Q$ range. Therefore for $340 \mathrm{~K}$ the measurements were restricted to $0.86 \leqslant Q \leqslant 1.78 \AA^{-1}$ and for $320 \mathrm{~K}$ to $1.37 \leqslant Q$ $\leqslant 1.78 \AA^{-1}$. Typical measuring times were $14 \mathrm{~h}$. Perpendicular transmissions of 0.79 and 0.77 were measured for $\lambda$ $=5.6 \AA$ and $\lambda=8 \AA$, respectively. For each momentum transfer the instrumental resolution function was determined from the elastic scattering of the sample at $10 \mathrm{~K}$. The instrumental background from the cryofour and the $\mathrm{Al}$ container were measured separately with measuring times similar to those employed for the sample. It was thereafter subtracted from the experimental spectra using the appropriate transmission factors. The instrumental resolution effects were finally removed by dividing the background corrected spectra by the resolution function.

\section{RESULTS}

\section{A. Validation: Static structure factor}

To validate the structure obtained in the simulation, the neutron scattering static structure factor $S(Q)$ for the fully protonated sample was calculated directly from the atomic coordinates. A large number of frames throughout the trajectories were considered for doing the average. Figure 2 compares the results from the simulations with those obtained from measurements on the sample with spin polarization analysis. These were performed in the $Q$ range from 0.7 to $3.4 \AA^{-1}$ by means of DNS spectrometer(FZ Jülich, Germany). The small coherent contribution to the scattering from fully protonated samples poses difficulties to this kind of measurements and is responsible for the poor quality of the collected data. However, within the uncertainties, it can be followed that the agreement observed between simulations and experiment validates the structure at intermolecular and intramolecular levels obtained in the simulation cell and gives support to the assumption that the simulations consti- 


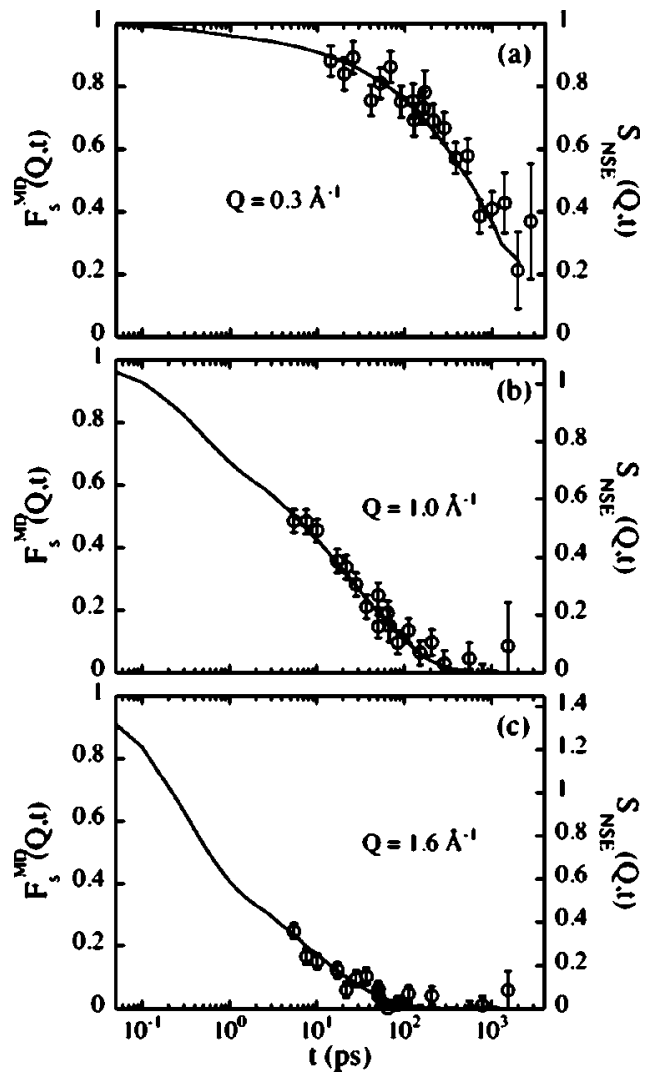

FIG. 3. Incoherent intermediate scattering function corresponding to all protons in PVE at $418 \mathrm{~K}$. Simulation results (lines) and IN11C results (symbols) at the $Q$ values indicated.

tute a reliable representation of the actual system. Although a complete structural characterization of the simulation cell is beyond the aim of this paper, we can anticipate that there is also a good agreement between the calculated static structure factors corresponding to a partially deuterated and a fully deuterated PVE samples and those measured in real samples by means of DNS. Such results will be published elsewhere.

\section{B. Dynamic results: Experiment versus simulations}

The decay of the proton self-motion correlation function is best centered in the IN11C dynamical window for the highest temperature investigated, $418 \mathrm{~K}$. For this temperature and three $Q$ values Fig. 3 compares the experimental NSE results with the intermediate scattering function calculated for all hydrogens in the PVE simulated cell. To do the comparison, for the two higher $Q$ values the NSE data have been plotted in a scale which differs from that of the simulations the more, the higher $Q$ is [8\% for $Q=1 \AA^{-1}$ in Fig. 3(b) and $45 \%$ for $Q=1.6 A^{-1}$ in Fig. 3(c)]. We note that after these corrections for the NSE amplitudes the agreement between both sets of data is strikingly good. In the following we will rationalize the need of such amplitude corrections and use the simulation results for a consistent renormalization of the experimental data.

As explained in the experimental section, the normalization of the NSE signal [Eq. (14)] is affected by the bandpass of the spectrometer. The values deduced from the measurements are not normalized to the full integral over all frequen-

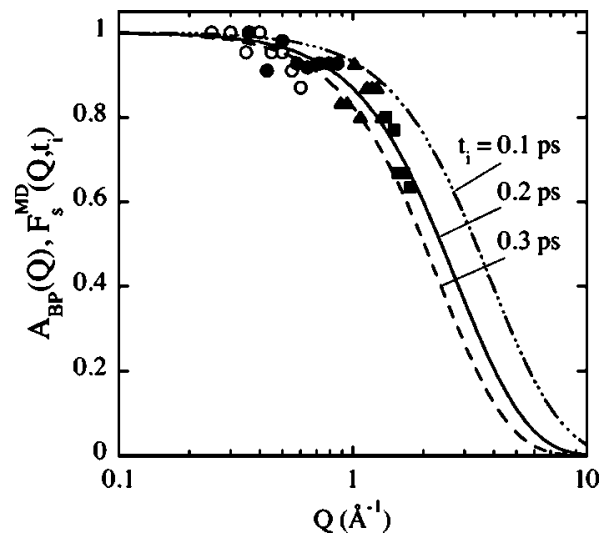

FIG. 4. Momentum transfer dependence of the effect of the bandpass on the amplitude of the NSE spectra obtained from the comparison with the MDsimulation results: $\lambda=8 \AA$ (empty circles) and $\lambda=5.6 \AA$ with $\theta=32^{\circ}$ (solid circles), $60^{\circ}$ (full triangles), and $90^{\circ}$ (full squares) for the central detector. The solid lines show the value of the intermediate scattering function $F_{s}(Q, t)$ for all protons in the sample calculated from the MD simulations for the different values of the time indicated.

cies but the integral is taken only over the bandpass (BP) of the spectrometer. This implies that the measured NSE signal differs from the properly normalized scattering function in a factor

$$
A_{B P}(Q)=\frac{\int_{B P} I(\theta, \omega) d \omega}{\int_{-\infty}^{+\infty} I(\theta, \omega) d \omega},
$$

where $I(\theta, \omega)=I_{c o h}(Q) \widetilde{S}(Q, \omega)-\frac{1}{3} I_{i n c} S_{s}(Q, \omega)$. This effect thus translates into an effectively smaller DWF. In the case of our protonated sample, as $I_{i n c} \gg I_{c o h}(Q)$, this expression becomes

$$
A_{B P}(Q)=\int_{B P} S_{S}(Q, \omega) d \omega
$$

since $\int_{-\infty}^{+\infty} S_{s}(Q, \omega) d \omega=1$ by definition.

At the used wavelengths the IN11C bandpass allows for energy transfers up to about $2 \mathrm{meV}$. Thus the normalization would include the so called fast processes observed at energies below about $2 \mathrm{meV}$ but excludes the ordinary vibrations. With increasing $Q$ and temperature, in the inelastic region the intensity of the scattering function due to vibrations increases, leading to higher deviations from the proper normalization.

This is, in fact, the trend observed for the amplitudes of the NSE data when compared with the simulation results. In the latter case, the uncertainties involved in the calculation of the static value of $F_{s}(Q, t)$ are very small. Therefore, the simulation results may be taken as a reference for determining the actual amplitudes of the experimentally deduced $F_{s}(Q, t)$. Under this assumption, we have estimated the bandpass correction factors $A_{B P}(Q)$ as those allowing to match the NSE data with the simulation results,

$$
A_{B P}(Q) S_{N S E}(Q, t) \leftrightarrow F_{s}^{M D}(Q, t) .
$$

The so obtained values for $A_{B P}(Q)$ are plotted in Fig. 4 for the different spectra measured at $418 \mathrm{~K}$. As commented above, the corrections become more and more significant 


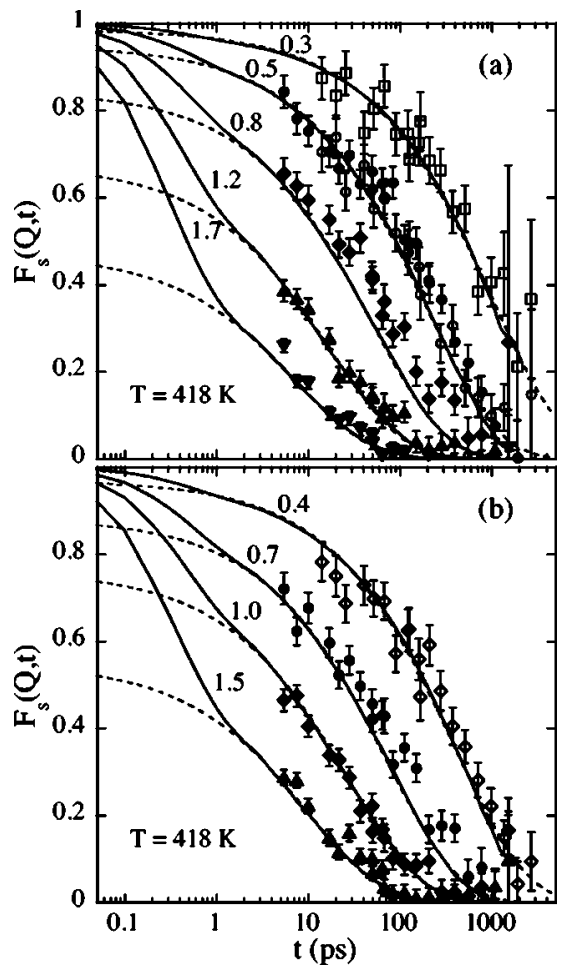

FIG. 5. Incoherent intermediate scattering function corresponding to all protons in PVE at $418 \mathrm{~K}$ : Simulation results (lines) and IN11C results (empty symbols stand for measurements with incident wavelength $\lambda=8 \AA$, and solid symbols for $\lambda=5.6 \AA$ ) at the $Q$ values indicated. The amplitudes of the experimental have been corrected for the bandpass of the spectrometer using the MD-simulation results at $t=0.3 \mathrm{ps}$. The dotted lines correspond to KWW descriptions of the second decay with $\beta=0.55$.

with increasing $Q$. On the other hand, the uncertainty in the determination of these values is quite high, as can be noticed from the scattering of the data.

In order to correct the NSE amplitudes with a meaningful criterion we have made use of the simulation results. The integral in Eq. (16) may be related to the value of the Fourier transform of the scattering function in an "effective bandpass time" $t_{B P}, F_{s}\left(Q, t_{B P}\right)$. This time should be of the order of the inverse of the bandpass frequency, $t_{B P} \approx \hbar / \hbar \omega_{B P}$ $\approx 0.3$ ps. Figure 4 displays the $Q$ dependence of $F_{s}(Q, t)$ as obtained from the simulations for $0.1,0.2$ and 0.3 ps and 418 $\mathrm{K}$. As can be appreciated from this figure, the values of $A_{B P}(Q)$ determined above are compatible with the curves corresponding to $F_{s}(Q, t=0.2 \mathrm{ps})$ and $F_{s}(Q, t=0.3 \mathrm{ps})$. We note that these values are very close to the estimated value for $t_{B P} . F_{s}(Q, t=0.3 \mathrm{ps})$ can thus be considered as a good approximation for the corrections of the NSE amplitudes for the bandpass. We note that this result gives additional support to the simulations.

Analogous comparisons for the other temperatures investigated led to the same estimate $t_{B P} \approx 0.3 \mathrm{ps}$. We then took this criterion for correcting the experimental amplitudes and the IN11C spectra were multiplied by $F_{s}^{M D}(Q, t$ $=0.3 \mathrm{ps}$ ). The results are shown in Fig. 5 for different $Q$ values at $418 \mathrm{~K}$ in comparison with the simulation results. For $Q=0.5 \AA^{-1}$, NSE results obtained by the two different incoming wavelengths are shown. Taking into account the experimental difficulties for measuring incoherent scattering

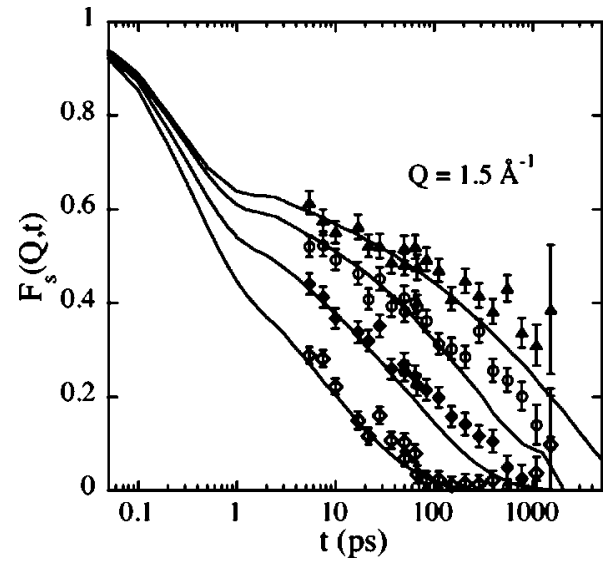

FIG. 6. Incoherent intermediate scattering function corresponding to all protons in PVE at $1.5 \AA^{-1}$ and $320 \mathrm{~K}, 340 \mathrm{~K}, 368 \mathrm{~K}$, and $418 \mathrm{~K}$ (top to bottom): Simulation results (lines) and IN11C results (symbols).

with NSE techniques, we emphasize the remarkable quality of the data. Also impressive is the agreement between experiments and simulations. We note that both sets of curves show very similar functional forms and also very close characteristic times. This implies that for $418 \mathrm{~K}$ the simulations account well for the $Q$ dependence of the scattering function and, moreover, reproduce well the overall time scale. Here there is no need to introduce a temperature shift in order to match the experimental data, as it was in the case of polyisoprene. There, a difference of about $40 \mathrm{~K}$ was reported between experiments and MD simulations, ${ }^{4}$ even though we have used in both cases the same force field (PCFF) for the simulations. Many possible reasons can be envisaged for this discrepancy, as, for instance, a more realistic extrapolated density in PVE than in the case of PI. The microstructure of the polymer simulated can also be closer to reality for PVE. It is also noteworthy that in the NVT simulation runs described in this work we have carefully checked that the pressure of the cell was, in average, close to the atmospheric pressure.

In addition, as can be seen in Fig. 6, the simulations also reproduce the experimentally observed temperature dependence of the proton dynamics. There for $Q=1.5 \AA^{-1}$ the spectra measured at the different temperatures are compared with their simulated counterparts. Within the experimental uncertainties the matching of both series of data can be considered as rather good. From this, thorough comparison between simulations and experiment, we can conclude that the simulations represent in a realistic way the dynamics of the real sample, at least as it is monitored by the self-motion of the protons, and at times above $\approx 5 \mathrm{ps}$. In the following we will focus on the results corresponding to this dynamical region, where the $\alpha$ relaxation is the main dynamic process leading to the decay of the correlations in the system. Here we will focus only on the $Q$ dependence of $F_{s}(Q, t)$. Therefore, we will take into account only the data corresponding to the highest temperature investigated, $418 \mathrm{~K}$, which show best statistics.

As can be seen in Fig. 5, the slow decay of $F_{s}(Q, t)$ above $t \approx 5 \mathrm{ps}$, which is controlled by the $\alpha$ process, can perfectly be described in terms of the well known KWW or 


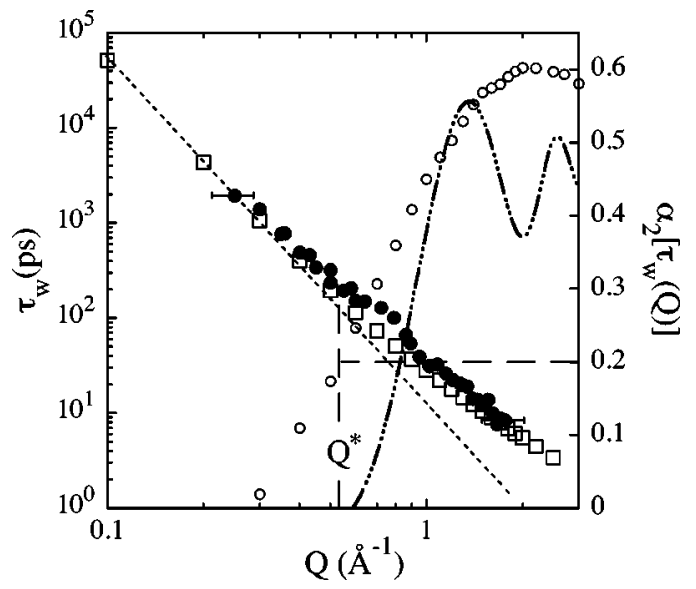

FIG. 7. $Q$ dependence of the characteristic time at $418 \mathrm{~K}$ obtained fixing the $\beta$ value to 0.55 for all the protons in PVE: results from the MD simulation (squares) and from IN11C (full circles). The dashed line shows the expected $Q$ dependence from the Gaussian approximation. The empty circles show the value of the non-Gaussian parameter evaluated at $t=\tau_{w}(Q)$ for each $Q$ value. The static structure factor $S(Q)$ is represented on the linear scale in arbitrary units. As representative examples, for the lowest and highest $Q$ values experimentally investigated the horizontal error bars show the uncertainty in the $Q$ value due to the width of the wavelength distribution used on IN11C.

stretched exponential function [Eq. (1)]. The fitting curves shown in Fig. 5 correspond to a fixed value of the shape parameter $\beta=0.55$. The good quality of the description achieved allows to take this constant value as a good approximation for the spectral shape at $418 \mathrm{~K}$. We note that in the description of the slow decay of $F_{s}(Q, t)$ by Eq. (1) the fast initial decay of the function is just parametrized by the DWF [Eq. (2)] characterized by the time-independent mean squared displacement $\left\langle u^{2}\right\rangle$. From the fit of Eq. (1) to the simulations results for $t \geqslant 5$ ps and the subsequent description of the amplitude $A(Q)$ by Eq. (2), a value of $0.77 \AA^{2}$ has been deduced for $\left\langle u^{2}\right\rangle$.

Since the determination of the $\beta$ value [and also of the amplitude $A(Q)]$ is subjected to a much higher uncertainty when analyzing the NSE data, and taking into account the fairly good agreement between experiment and simulation results, for a direct comparison of the time scales the IN11C curves were fitted by means of Eq. (1) with $\beta$ as determined from the simulations, $\beta=0.55$. The characteristic times obtained from this fit for both sets of data are plotted in Fig. 7, showing an excellent agreement. Only in a narrow $Q$ region around $0.7 \AA^{-1}$ the time scale as obtained from the NSE measurements is slightly larger than that deduced from the simulations. This sort of "bump" in the experimental data around $0.7 \AA^{-1}$ is a reminiscence of coherent contributions. A similar effect was also found in Ref. 4 for NSE results on polyisoprene main chain protons. Close to the main peaks of the structure factors, the collective dynamics tends to slow down (de Gennes narrowing ${ }^{27}$ ), and coherent contributions there lead to effectively larger times for the measured decay function. We note that the bump observed in the dynamic measurements appears at lower $Q$ values than the static correlation peak. This effect has already been experimentally observed on fully deuterated polybutadiene ${ }^{28}$ and PIB (Ref. 29) samples by NSE. In this last case, MD simulations cor- roborate such a shift. ${ }^{3}$ The position of the maximum characteristic time moves towards lower $Q$ values with increasing temperature.

After this exhaustive check of the capability of our MD simulations for reproducing the experimental observations on the real sample, we can conclude that they indeed offer a strikingly good mimic of the real polymer, at least for the problem we are dealing with in this work: the self-motions of the protons in the $\alpha$-relaxation regime. We note that, in addition, the structural properties are well reproduced and the results on the microscopic region show consistency with the NSE amplitudes. Such a good agreement between MDsimulation results and dynamic data has never been reported so far for a glass forming polymer. In fact, as most of the simulations are performed either on toy (bead and spring) model $\mathrm{s}^{30}$ or on united atom models, ${ }^{31}$ the direct comparison with microscopic measurements on real systems at atomic level (interchain and intrachain length scales) is not possible. The reasonable agreement found here gives us confidence that those results obtained from simulation which are not easily accessible by the current experimental techniques will also be reasonably realistic. This is, in fact, the ultimate goal of any simulation exercise: once a realistic system is simulated, take advantage of it to go beyond the experimental possibilities. In addition to the incoherent scattering function, here we will specially focus on the non-Gaussian parameter $\alpha_{2}(t)$ and the mean square displacement $\left\langle r^{2}(t)\right\rangle$ for protons, mainly at time scales above the picosecond range. This is where the validation has been performed. The MDsimulation results on these functions at shorter times will also be presented and shortly discussed. The basis to rely on these results is the somehow indirect crosscheck with the NSE results resting on the consistency of the bandpass corrections.

\section{DISCUSSION}

\section{A. Crossover from Gaussian to non-Gaussian behavior}

For a large number of glass forming polymers ${ }^{1,2,32}$ in the $Q$ range usually covered by neutron scattering techniques $\left(0.2 \leqslant Q \leqslant 1.5 \AA^{-1}\right)$ it has been established that the $Q$ dependence of the characteristic time for the self-motion of the protons is determined by the stretching exponent $\beta$ as described by Eq. (3). This relation implies that the incoherent scattering function $G_{s}(r, t)$ and $F_{s}(Q, t)$ show the Gaussian forms given by Eqs. (9) and (11). Recent MD simulations on PI (Ref. 3) have addressed the question of the limits of Gaussian behavior for the self-motions of the main chain protons in such polymer-methyl group protons were not considered in order to avoid the influence of the fast motions related to the methyl group rotations. ${ }^{33,34}$ Clear deviations from Eq. (3) were found for $\tau_{w}(Q)$ at $Q$ values above $Q^{\star}$ $\approx 1.3 \AA^{-1}$. For $Q \geqslant Q^{\star}$ a clearly weaker $Q$ dependence for $\tau_{w}(Q)$ developed. This value of $Q^{\star}$ coincides with that of the first maximum of the static structure factor $Q_{\max }$ in that polymer. The presence of such deviations was thereafter experimentally confirmed $\mathrm{d}^{4,35}$ by extending the $Q$ range of the $F_{s}(Q, t)$ study up to $\approx 5 \AA^{-1}$. 


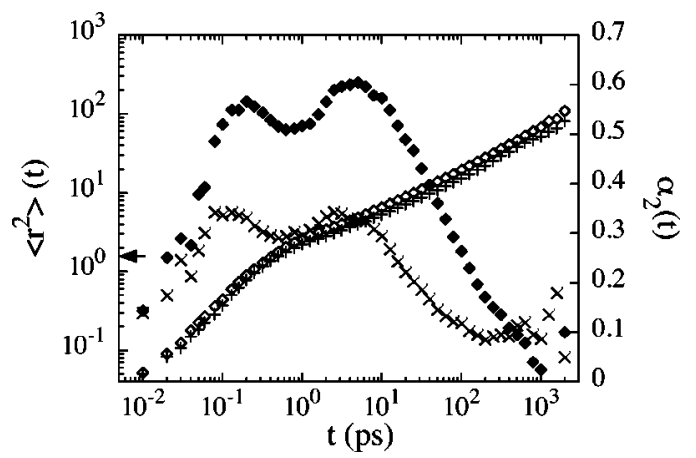

FIG. 8. Time evolution of the mean squared displacement $\left\langle r^{2}\right\rangle$ (empty diamonds) and the non-Gaussian parameter $\alpha_{2}$ (full diamonds) calculated from the MD simulations for all hydrogens in PVE. The analogous results for the main chain protons in PI at $413 \mathrm{~K}(\times,+)$ are shown for comparison Ref. 3. The horizontal arrow indicates the value of $2\left\langle u^{2}\right\rangle$ deduced from the description by Eq. (2) of the amplitudes deduced from the fit of Eq. (1) to the $F_{s}(Q, t)$ obtained from the PVE simulations at $t \geqslant 5 \mathrm{ps}$.

What do we find for the case of PVE? Just at first sight, unusually strong deviations from Gaussian behavior can be envisaged. Figure 7 compares the $Q$ dependence of the obtained characteristic times for all protons in the sample with the Gaussian prediction (power law indicated by the dotted line). In this logarithmic representation the time bends systematically upwards with increasing $Q$, deviating from the Gaussian relation already at $Q^{\star} \approx 0.5 \AA^{-1}$. This behavior is found for the times deduced from both, the experimental and the MD-simulation results.

At this point we begin to exploit the unique information provided by the MD simulations in real space. For instance, we may directly compare the incoherent scattering function with the Gaussian prediction [Eq. (11)]. This is not possible from the NS experiments since the knowledge of the proton mean squared displacement $\left\langle r^{2}(t)\right\rangle$ is required. Figure 8 displays the resulting $\left\langle r^{2}(t)\right\rangle$ for PVE protons at $418 \mathrm{~K}$ (diamonds), and Fig. 9 the consequent expectation from the Gaussian approximation for $F_{s}(Q, t), F_{s}^{\text {Gauss }}(Q, t)$ [Eq. (11)] (circles). The complete function $F_{s}(Q, t)$ shows a retardation in the decay with respect to $F_{s}^{\text {Gauss }}(Q, t)$ that starts to be

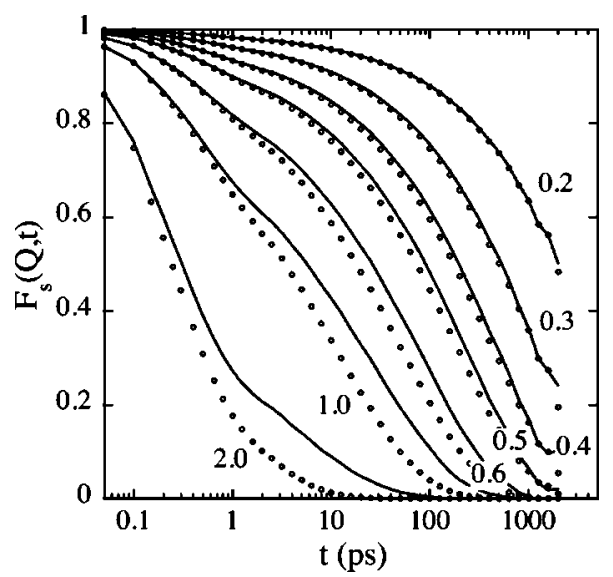

FIG. 9. Comparison between the incoherent scattering function $F_{s}(Q, t)$ (lines) and the result of the Gaussian approximation (circles) for the indicated different $Q$ values (given in $\AA^{-1}$ ). detectable for $Q$ values as small as $0.4 \AA^{-1}$ and becomes the more noticeable, the higher the $Q$.

It is important to emphasize the extremely low value observed for $Q^{\star}$ in this polymer. Contrarily to the case of PI, the crossover towards non-Gaussian behavior for PVE occurs at $Q$ values much smaller than the static structure factor peak (see Fig. 7), $Q^{\star} \ll Q_{\max }$. For PI, it was shown that the crossover at $Q^{\star}$ was directly related with the value of the non-Gaussian parameter at the characteristic time for the self-motion at each $Q, \alpha_{2}\left[t=\tau_{w}(Q)\right]$. The crossover seems to take place when this function takes values of about $0.2, \ldots, 0.25$. This feature was found in a wide temperature range and at a constant temperature for different densities in that polymer. ${ }^{36}$ Starting from the non-Gaussian parameter $\alpha_{2}(t)$ obtained from our simulations for PVE protons [see Fig. 8 (full diamonds)], we have evaluated the function $\alpha_{2}\left[t=\tau_{w}(Q)\right]$. This is shown in Fig. 7 as a function of $Q$. Again for this polymer we observe that $Q^{\star}$ is located when $\alpha_{2}\left[t=\tau_{w}(Q)\right]$ takes values of the order of 0.2. Thus, it seems that the relation

$$
\alpha_{2}\left[t=\tau_{w}\left(Q^{\star}\right)\right] \approx 0.2
$$

roughly determines the value of $Q^{\star}$.

Now let us consider in more detail $\left\langle r^{2}(t)\right\rangle$ and $\alpha_{2}(t)$ for PVE protons. Figure 8 compares these functions at $418 \mathrm{~K}$ with the corresponding results obtained for the main chain protons of PI at $413 \mathrm{~K}^{3}$ We have chosen this temperature for the comparison because there the values of $\left\langle r^{2}(t)\right\rangle$ are very much the same for the protons of both polymers, i.e., the average proton mobilities are very similar. For both polymers $\left\langle r^{2}(t)\right\rangle$ displays three typical dynamic ranges: (i) a microscopic regime until about $0.8-1 \mathrm{ps}$; (ii) a crossover regime until about $10 \mathrm{ps}$; and (iii) a sublinear time dependence which extends until the limit of the simulations. We may comment at this point that the parameter $\left\langle u^{2}\right\rangle$ used in Eq. (2) within Eq. (1) gives account for the decrease of the scattering function due to the increase of $\left\langle r^{2}(t)\right\rangle$ in the first fast regime. From the comparison between Eqs. (1) and (2) and Eq. (11), in the Gaussian approximation $2\left\langle u^{2}\right\rangle$ would provide an estimation of the value reached by $\left\langle r^{2}(t)\right\rangle$ just before the $\alpha$ relaxation sets in (see horizontal arrow in Fig. 8). Turning now to the non-Gaussian parameter, we observe in the same figure that for both polymers $\alpha_{2}(t)$ has a double peak structure. The short-time maximum corresponds to the microscopic regime of $\left\langle r^{2}(t)\right\rangle$ and the other is centered in the crossover regime of $\left\langle r^{2}(t)\right\rangle$. This second peak of $\alpha_{2}(t)$ shows a similar behavior to that observed in computer simulations of simple Lennard-Jones systems ${ }^{5}$ or in the experiments with colloidal glass-forming systems. ${ }^{37}$ Once the sublinear behavior of $\left\langle r^{2}(t)\right\rangle$ is well established, $\alpha_{2}(t)$ decreases to its long time limit, zero. Though the qualitative behavior is the same for both PI and PVE and the values of $\left\langle r^{2}(t)\right\rangle$ are practically indistinguishable, the strong deviations from Gaussianity in PVE lead to much higher values for $\alpha_{2}(t)$. PVE protons exhibit $\alpha_{2}(t)$ values twice as large as those of PI main chain protons. What could be the origin of such strong deviations from Gaussian behavior in PVE? 


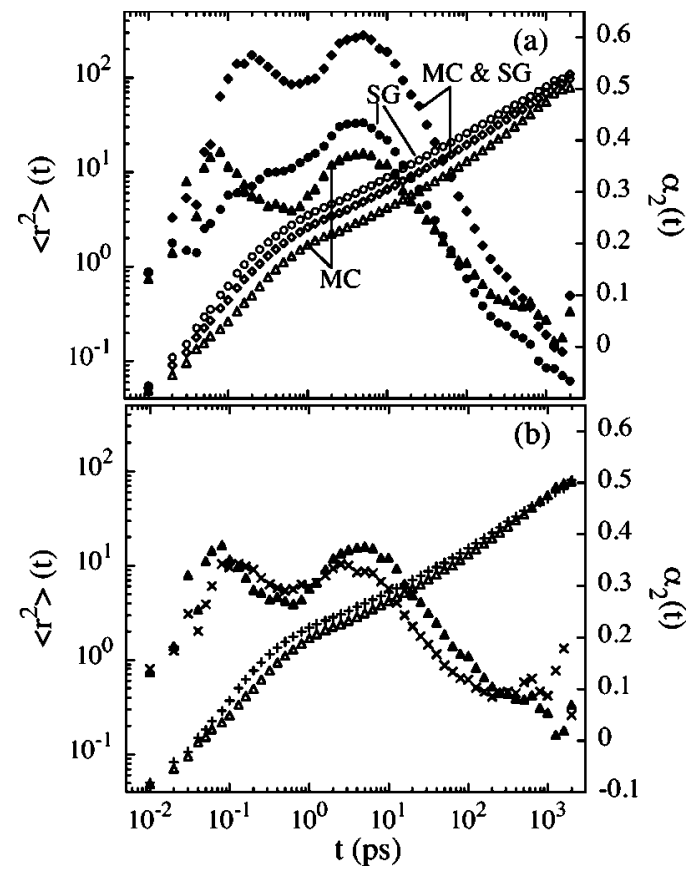

FIG. 10. Time evolution of the mean squared displacement $\left\langle r^{2}\right\rangle$ (empty symbols) and the non-Gaussian parameter $\alpha_{2}$ (full symbols) calculated from the MD simulations for the main chain hydrogens (triangles), side group hydrogens (circles), and all hydrogens (diamonds) in PVE (a). The results corresponding to the main chain hydrogens are compared in (b) with the analogous in PI at $413 \mathrm{~K}(\times,+)$.

\section{B. Side group dynamics versus main chain dynamics}

As can be appreciated in the scheme of the monomer depicted in Fig. 1, PVE contains a big side group with the same number of atoms as those included in the main chain. If the dynamics of side group hydrogens is different from that corresponding to the main chain, which seems to be quite plausible, we should expect a strong enhancement of nonGaussianity. We thus have considered separately main chain (MC) and side group (SG) protons (see Fig. 1). The calculated $\left\langle r^{2}(t)\right\rangle$ and $\alpha_{2}(t)$ for both kinds of protons are shown in Fig. 10(a) together with the results corresponding to all protons previously discussed. For $\left\langle r^{2}(t)\right\rangle$ the qualitative behavior is similar for MC and SG protons, showing the same dynamic ranges as described above for all the protons. However, we note that (i) SG protons display larger displacements than MC protons already from very fast times, (ii) the plateau characteristic for the cage effect in the crossover region is more pronounced for MC protons, and (iii) the sublinear regime of these atoms exhibits a stronger slope. At the longest times explored by the simulation, the value of $\left\langle r^{2}(t)\right\rangle$ is very similar for both kinds of protons. This observation is natural, since the atoms within a monomer are linked among them. For sufficiently long times in the supercooled liquid state, the differences in the displacements have to be negligible. Obviously, the curve corresponding for all protons shows an intermediate behavior, since it is the average of both functions. Turning to the non-Gaussian parameter, we observe that the first peak for MC protons is centered at relatively short times and well separated from the second peak. The intensity of both peaks is very similar. On the contrary, SG protons exhibit a quite weak first peak as compared with the second one, so that it appears like a shoulder of the main maximum. We note that, though the second maximum takes higher values for SG protons, its position is just the same for both SG and MC protons. It is centered in the early stages of the sublinear regime leading to the $\alpha$ relaxation. The non-Gaussian processes reflected by this peak are responsible for the deviations from Gaussianity observed at $Q \geqslant Q^{\star} .{ }^{3}$ On the other hand, the first peaks are related to very fast motions of the protons-in fact, to $\mathrm{C}-\mathrm{H}$ librations ${ }^{3}$ - and it can be expected that they do not play a relevant role in the dynamic range typical for the $\alpha$ relaxation. The result of averaging the dynamics of the two kinds of atoms for $\alpha_{2}(t)$ is straightforward to derive: as the number of MC and SG hydrogens in the system is same, the averaged even moments can be calculated as

$$
\left\langle r_{M C \& S G}^{2 n}(t)\right\rangle=\frac{1}{2}\left[\left\langle r_{M C}^{2 n}(t)\right\rangle+\left\langle r_{S G}^{2 n}(t)\right\rangle\right] .
$$

Now, considering this result and the definition of $\alpha_{2}(t)$ [Eq. (13)], we obtain

$$
\begin{aligned}
\alpha_{2}^{M C \& S G}(t) & \\
= & 2 \frac{\left\langle r_{M C}^{2}(t)\right\rangle^{2}\left(\alpha_{2}^{M C}(t)+1\right)+\left\langle r_{S G}^{2}(t)\right\rangle^{2}\left[\alpha_{2}^{S G}(t)+1\right]}{\left[\left\langle r_{M C}^{2}(t)\right\rangle+\left\langle r_{S G}^{2}(t)\right\rangle\right]^{2}}-1 .
\end{aligned}
$$

As expected, the self-correlation function for all hydrogens shows much more severe deviations from Gaussian behavior than those observed for each kind of atoms. In Fig. 10(a) we can see this effect. In fact, if we only consider the main chain protons in PVE, their corresponding $\left\langle r^{2}(t)\right\rangle$ and $\alpha_{2}(t)$ are very similar to those of main chain PI protons, as it is demonstrated in Fig. 10(b). Therefore we can certainly attribute the strong deviations from the Gaussian approximation found in PVE to the heterogeneity in the dynamics of its protons. Being a polymer with a relatively large number of atoms located in lateral groups, the differences in the dynamics within the main chain and the side groups are reflected in the breakdown of the Gaussian approximation at very low $Q$ values.

Thus, side group and main chain PVE protons show quite similar values for $\alpha_{2}(t)$ in the region of the decaging, which, by the way, are close to those of PI main chain protons. These values are non-negligible and for the reported case of PI they led to significant deviations in the $Q$ dependence of $\tau_{w}(Q)$ with respect to Eq. (3) at $Q^{\star} \approx 1.3 \AA^{-1}$. In order to scrutinize the $Q$ dependence of the characteristic time for MC and SG protons of PVE, we have calculated the scattering functions for both kind of protons. They are shown in Fig. 11 for several $Q$ values together with $F_{s}(Q, t)$ for all the protons. For all $Q$ values the function corresponding to the SG protons decays faster than that of the MC protons. As expected, the curve obtained for all hydrogens shows an intermediate behavior. Fits with KWW functions [Eq. (1)] lead to higher values for the stretching parameter $\beta$ for MC protons than for SG protons: we obtain the very good fit of the slow decays shown in Fig. 11 with $\beta=0.66$ and $\beta=0.51$ for MC and SG hydrogens, respectively (we remind that for all protons we found an intermediate value $\beta=0.55$ ). Such differences in the spectral shapes could be expected from the 


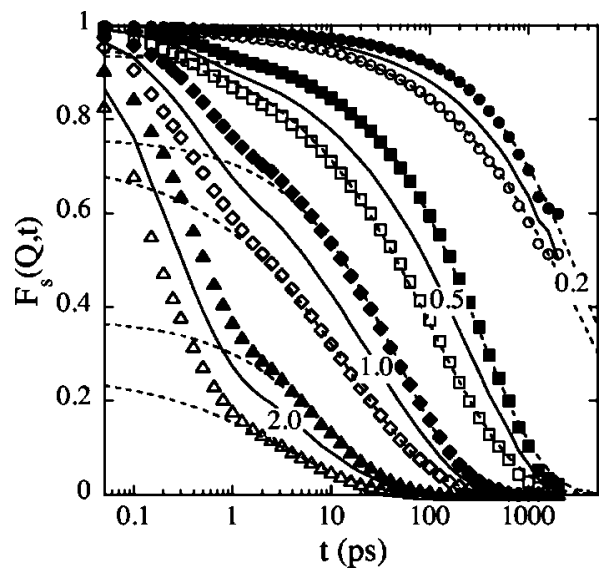

FIG. 11. MD-simulation results for the incoherent intermediate scattering function corresponding to the main chain hydrogens (full symbols), side group hydrogens (empty symbols), and all hydrogens (lines) at the $Q$ values indicated. The dotted lines correspond to KWW descriptions of the second decay with $\beta=0.51(\mathrm{SG})$ and $\beta=0.66(\mathrm{MC})$.

inspection of the slopes of the mean squared displacement in Fig. 10(a), since the sublinear increase with time of $\left\langle r^{2}(t)\right\rangle$ in the $\alpha$-relaxation regime is given by $\left\langle r^{2}(t)\right\rangle \propto t^{\beta}$. This can be deduced assuming the Gaussian approximation (which for sufficiently long times after the plateau is reasonably well fulfilled) for a KWW-like intermediate scattering function. ${ }^{1}$ Concerning the mean squared displacement associated with the first fast decay, $\left\langle u^{2}\right\rangle$, we find $\left\langle u_{M C}^{2}\right\rangle=0.76 \AA^{2}$ and $\left\langle u_{S G}^{2}\right\rangle=1.02 \AA^{2}$. This difference can also be intuitively rationalized by looking at the values of $\left\langle r^{2}(t)\right\rangle$ for both kinds of atoms in the region $t \approx 1$ ps in Fig. 10(a).

The deduced values for $\tau_{w}(Q)$ are displayed in Fig. 12 for both types of atoms. They show deviations from their corresponding Gaussian power laws at different $Q$ values: $Q_{M C}^{\star} \approx 1 \AA^{-1}$ while $Q_{S G}^{\star} \approx 0.6 \AA^{-1}$. Somehow, the result for the SG hydrogens may be surprising, since we have seen in Fig. 10(a) that the values of $\alpha_{2}(t)$ are, though slightly higher for SG than for MC protons in the region of the second peak, not so much different. We observe in the same figure that

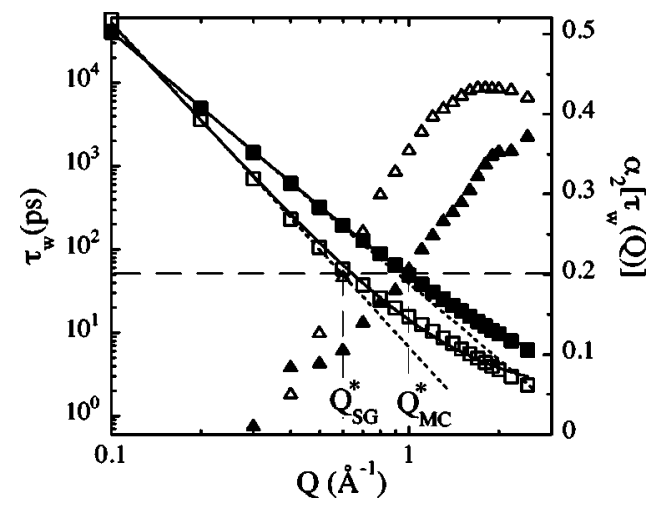

FIG. 12. Momentum transfer dependence of the characteristic time deduced from the MD simulations for the main chain hydrogens (full squares) and the side group hydrogens (empty squares) in PVE. The non-Gaussian parameter calculated at $t=\tau_{w}(Q)$ is also plotted (empty triangles, main chain hydrogens; full triangles, side group hydrogens). The dotted lines show the Gaussian prediction and the solid lines the description in terms of the anomalous jump diffusion model. $\alpha_{2}(t)$ assumes values close to 0.2 just at the same time $t$ $\approx 50 \mathrm{ps}$ for both kinds of atoms. This is just the key for understanding the behavior found: as the mobility of SG protons is higher than that of MC protons, their characteristic time for a given $Q$ value is faster. This implies that the same value of $\tau_{w}$ is observed at lower $Q$ values for SG hydrogens. In fact, we find $Q^{\star}$ in Fig. 12 just searching the $Q$ value at which the characteristic time is $\tau_{w} \approx 50 \mathrm{ps}$. Thus again in this case the condition proposed for identifying $Q^{\star}$ [Eq. (18)] works. We have included in Fig. 12 the values of $\alpha_{2}[t$ $\left.=\tau_{w}(Q)\right]$ corresponding to both $\mathrm{MC}$ and SG protons, showing how well the proposed condition is fulfilled. These results corroborate that the deviations from Gaussian behavior are determined by the quantity $\alpha_{2}\left[t=\tau_{w}(Q)\right]$.

As it was discussed in Ref. 4, different theoretical approaches can be invoked to understand the crossover form Gaussian to non-Gaussian behavior of the proton selfcorrelation function in the $\alpha$ regime of glass forming polymers. In Ref. 4, we also developed a simple picture which, in principle, seems to be compatible with the framework of the mode coupling theory. ${ }^{38}$ The proposed model is a generalization of the well known jump diffusion model ${ }^{39,40}$ to the case of a sublinear diffusion. This simple interpretation is based on the existence of a distribution of discrete jumps underlying the atomic motions in the $\alpha$ process. In this frame, at low $Q$ corresponding to large distances, we deal with a Gaussian sublinear diffusion process. At higher $Q$ (shorter distances) the Gaussianity breaks down due to the discrete character of the sublinear diffusion. A distribution of jump lengths $\ell$

$$
f(\ell)=\left(\ell / \ell_{o}^{2}\right) \exp \left(-\ell / \ell_{o}\right)
$$

results from the disorder characterizing glass forming polymers. $\ell_{o}$ is the preferred jump distance. In its range of applicability this very simple model was able to capture the main features of $\tau_{w}(Q)$ and $\alpha_{2}(t)$ for PI at $363 \mathrm{~K}$ and is found to give account for the main "universal features" reported in the literature for the behavior of the non-Gaussian parameter. ${ }^{4,41}$ In a recent work, ${ }^{36}$ we have shown for PI that also the temperature and density dependencies of $\alpha_{2}[t$ $\left.=\tau_{w}(Q)\right]$ are reproduced by this model. Now we can apply it to the case of PVE data. The characteristic time in this model is given by

$$
\tau_{w}=\tau_{o}\left[1+\frac{1}{Q^{2} \ell_{o}^{2}}\right]^{1 / \beta}
$$

where $\tau_{o}$ is a microscopic residence time. The fit of Eq. (22) to the characteristic times obtained for both MC and SG protons leads to the good descriptions shown in Fig. 12. The values obtained for $\ell_{o}$ and $\tau_{o}$ are $\ell_{o}^{M C}=0.39 \AA, \ell_{o}^{S G}$ $=0.73 \AA, \tau_{o}^{M C}=2.2 \mathrm{ps}$, and $\tau_{o}^{S G}=1.8 \mathrm{ps}$. The corresponding distributions of jump lengths are shown in Fig. 13. The SG hydrogens perform larger elemental jumps than the MC protons in their motion during the structural relaxation. This result is intuitively easy to understand. On the other hand, we note that the distribution of jump lengths for MC protons is 


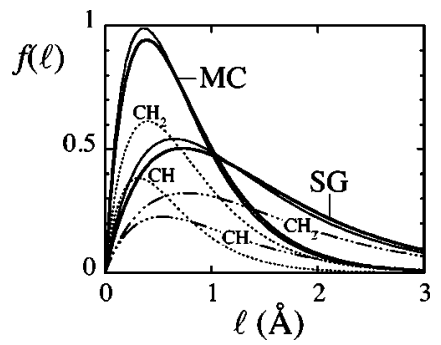

FIG. 13. Distributions of jump lengths deduced from the application of the anomalous jump diffusion model for main chain and side group protons of PVE (solid thick lines). Dotted (dashed-dotted) lines show the distributions obtained for the main chain (side group) methyne and methylene protons, weighted by the relative abundance of the corresponding kind of hydrogen in the monomer. The thin solid lines are the addition of the weighted distributions for methyne and methylene protons either in the main chain or in the side group.

very similar to that found for main chain protons in $\mathrm{PI}-$ there $\ell_{o}$ amounted to $0.42 \AA$. This result gives additional support to the proposed model.

\section{Differences between methyne and methylene dynamics}

Now exploiting further the possibilities of MD simulations we can also investigate the dynamics of hydrogen atoms belonging either to the $\mathrm{CH}_{2}$ (methylene) or $\mathrm{CH}$ (methyne) groups. Figure 14 shows the results obtained for $\left\langle r^{2}(t)\right\rangle$ and $\alpha_{2}(t)$ corresponding to the four types of protons we can distinguish in PVE. In the upper part of the figure the methyne hydrogens are considered, while below the results on the methylene protons are shown. The following features can be observed:

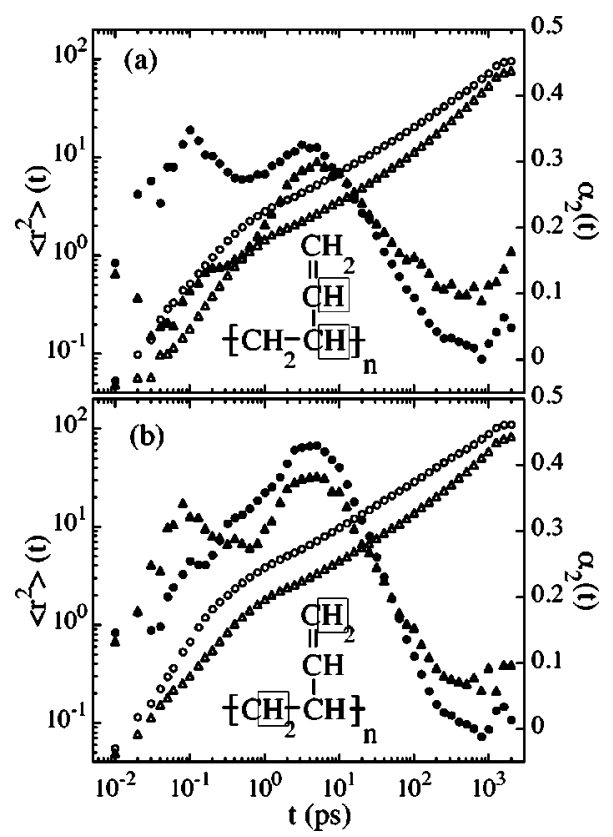

FIG. 14. Time evolution of the mean squared displacement $\left\langle r^{2}\right\rangle$ (empty symbols) and the non-Gaussian parameter $\alpha_{2}$ (full symbols) calculated from the MD simulations for the methyne hydrogens (a) and methylene hydrogens (b) in PVE. Triangles correspond to atoms located in the main chain and circles to those in the side groups. (i) Comparing the mean squared displacement we see that for both methyne and methylene protons, those in the main chain show a more reduced mobility than those in the side group. Moreover, the methyne proton is less mobile than the methylene protons when either $\mathrm{MC}$ or $\mathrm{SG}$ atoms are considered.

(ii) For the non-Gaussian parameter we can discuss the comparison for three time regions: in the microscopic regime the first peak is very similar for the methyne protons of the side group and the methylene protons in the main chain.

Both exhibit a well defined peak with similar value at the maximum; that is, centered at about $0.1 \mathrm{ps}$. This is, in principle, a somehow unexpected and astonishing result. The situation is quite different for the rest of the hydrogens: the first peak is a kind of shoulder of the main second peak. Now we concentrate in the time region where the second maximum is observed. For all hydrogens the position of this peak is very much the same, around 4 ps. However, the side group protons show higher maxima than main chain protons, and we note that the value at the maximum increases for methylene protons with respect to that for methyne protons. In fact, the maximum height is bigger for methylene main chain protons than for methyne side group protons. Finally, at large times, where the subdiffusive regime of the $\alpha$ relaxation is established, the value of $\alpha_{2}(t)$ is very similar for all side group protons, showing values very close to zero, while main chain protons seem to present a slightly positive value around $0.08, \ldots, 0.1$ in this regime.

Comparing these results with those reported for the different hydrogens in PI (see Fig. 7 of Ref. 3), we deduce that the behavior of $\alpha_{2}(t)$ observed for the double bond methyne hydrogens of PI is very similar to that of the methylene side group protons of PVE-also bound to a double bond. On the other hand, the methylene PI hydrogens show an intermediate behavior between methylene side group protons and methylene main chain protons in PVE.

As we can see, each type of hydrogen shows a particular dynamic behavior in the time regions corresponding to the microscopic regime, the cage and the establishment of the $\alpha$ process. In particular, the short-time regime of both $\left\langle r^{2}(t)\right\rangle$ and $\alpha_{2}(t)$ strongly depends on the kind of atom considered. This implies that the short time behavior observed has to be very different in a realistic polymer and in a simple model liquid, and such kind of details could never be resolved in simple model systems as bead-spring or united-atom polymer models.

In the light of these results we may ask what is the effect of the differences in the dynamics between methylene and methyne protons on the deviations from Gaussian behavior observed for MC and SG protons in the preceding section. To do this the scattering functions for each kind of proton have been calculated and analyzed in terms of KWW functions. The results can be seen for two $Q$ values and all types of protons in Fig. 15. We observe that for $Q=0.8 \AA^{-1}$ the functions decay with different characteristic times and show different stretching for the different protons. For a given time, the value of $F_{s}(Q, t)$ increases in the following order: SG methylene, SG methyne, MC methylene, and MC methyne hydrogens. For the lower $Q$ value shown in the figure, $Q$ 


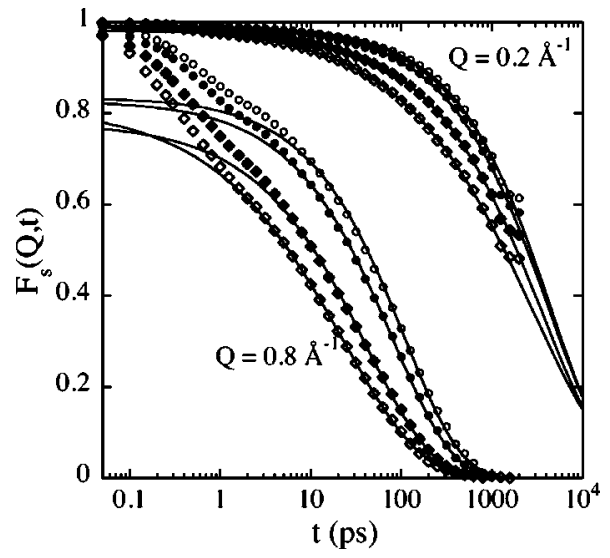

FIG. 15. Incoherent intermediate scattering function corresponding to all the different kinds of hydrogens in PVE: MC methyne (empty diamonds), MC methylene (full diamonds), SG methyne (empty circles), and SG methylene (full circles) at the $Q$ values indicated. The solid lines correspond to KWW descriptions of the second decay with the $\beta$ values shown in Table I.

$=0.2 \AA^{-1}$, the characteristic time as deduced from the extrapolation of the fitting curves becomes very similar for the different hydrogens. However, the decays at times shorter than $\tau_{w}$ can be perfectly distinguished, showing similar behavior to that described for the case of $Q=0.8 \AA^{-1}$. For the different protons, the values obtained for the $\beta$ parameter are found to vary from 0.51 to 0.70 and $\left\langle u^{2}\right\rangle$ ranges between 0.72 and $1.11 \AA^{2}$ (see Table I). The resulting time scales are shown in Fig. 16(a). As observed above, they become close together in the low $Q$ region. Towards high $Q$ they decrease following the Gaussian expectation with different slopes according to the different $\beta$ value obtained for each kind of proton. At higher $Q$ 's the times deviate from such behavior and show a tendency to merge in the high $Q$ limit. We note that the time when the deviations from the corresponding power law begin to be appreciated is, within the uncertainties, very similar for all curves: about $50 \mathrm{ps}$. This is again the time where all the non-Gaussian parameters take values close to $0.2, \alpha_{2}(t=50 \mathrm{ps}) \approx 0.2$ (see Fig. 14). We have plotted $\alpha_{2}\left[t=\tau_{w}(Q)\right]$ in Fig. 16(b) for all kinds of protons. The correlation between $\alpha_{2}\left[t=\tau_{w}(Q)\right] \approx 0.2$ and the deviations of $\tau_{w}(Q)$ from Eq. (3) can be seen from this figure.

Finally we can ask what are the resulting distributions of jump lengths when the anomalous jump diffusion model is considered for the different kinds of hydrogens. The fit of Eq. (22) to the time scales yields the curves shown in Fig. 16(a) as solid lines. Though the description is not perfect, taking into account the simplicity of the model, it can be considered as quite satisfactory. The resulting values for $\ell_{o}$

TABLE I. Parameters characterizing the shape and amplitude of the $\alpha$-relaxation decay of $F_{s}(Q, t)$, the jump distribution function, and the microscopic residence time for the different kinds of PVE protons.

\begin{tabular}{ccccc}
\hline \hline Hydrogen type & $\beta$ & $\left\langle u^{2}\right\rangle\left(\AA^{2}\right)$ & $\ell_{o}(\AA)$ & $\tau_{o}(\mathrm{ps})$ \\
\hline MC methyne & 0.70 & 0.72 & 0.32 & 1.87 \\
MC methylene & 0.66 & 0.80 & 0.40 & 2.24 \\
SG methyne & 0.60 & 1.10 & 0.54 & 1.99 \\
SG methylene & 0.51 & 1.11 & 0.76 & 1.71 \\
\hline \hline
\end{tabular}

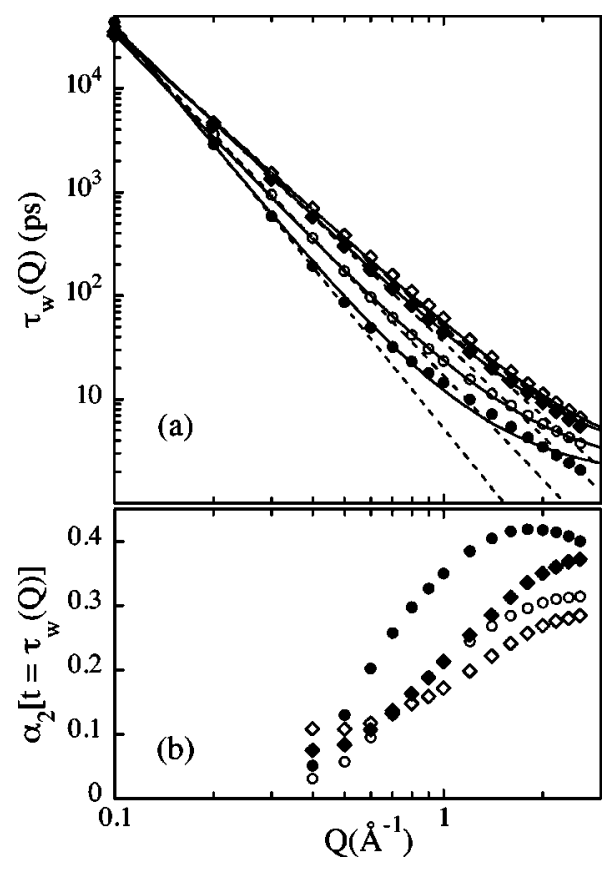

FIG. 16. Momentum transfer dependence of (a) the characteristic time for self motion and (b) the non-Gaussian parameter evaluated at $t=\tau_{w}(Q)$ for all the different kinds of hydrogens in PVE: MC methyne (empty diamonds), MC methylene (full diamonds), SG methyne (empty circles), and SG methylene (full circles). In (a) the dotted lines show the Gaussian prediction and the solid lines the description in terms of the anomalous jump diffusion model.

and $\tau_{o}$ are shown in Table I. As could be expected, the values for $\ell_{o}$ are quite close for both kinds of protons in the main chain, while in the side group the methyne hydrogen presents a smaller average jump length than the methylene protons (being both larger than those of main chain protons). We can see the corresponding distribution functions of jump lengths in Fig. 13. They have been weighted by the relative abundance of each type of proton in the system. The results obtained in the preceding section for MC and SG hydrogens are a sort of average of the corresponding distribution functions for methyne and methylene protons. On the other hand, the residence time $\tau_{o}$ for all kind of protons is very similar and close to 2 ps. Again these findings are reasonable. We can then conclude that even considering each kind of proton separately, there exist remaining deviations from Gaussian behavior in the hydrogen self-correlation function. These can be accounted for in the frame of the anomalous jump diffusion model proposed in Refs. 35 and 4, despite its simplicity.

\section{CONCLUSIONS}

By exhaustive comparison of the structure and the dynamics of the protons in the time region $5 \mathrm{ps} \leqslant t \leqslant 2 \mathrm{~ns}$ with neutron scattering results we have first of all demonstrated that our simulated sample provides a good representation of real PVE. Then we have established that the deviations from Gaussian behavior in the self-motion of protons are stronger for PVE than for other simpler polymers as PI or 1,4-polybutadiene. ${ }^{42}$ This bases both on the experimental NSE and on the MD-simulations results. This behavior has been explained taking into account the additional contribu- 
tions to non-Gaussianity from the different mobilities of the hydrogens in this polymer (in particular, main chain and side group hydrogens), which can be deduced from the MD simulations.

Therefore, we can conclude, first of all, that PVE-and likely other polymers - is an intrinsically heterogeneous system showing different dynamics for its different atomic constituents. Such dynamical heterogeneity is, however, inherent to the chemical composition and microstructural details of the system and, obviously, cannot be easily related to the heterogeneous behavior found in simple glass forming models as, for instance, Lennard-Jones systems.

On the other hand, even considering separately each type of proton, deviations from Gaussianity are found. We have shown that in all cases the crossover from Gaussian to nonGaussian dynamics takes place if the $Q$-dependent nonGaussian parameter at the corresponding characteristic time reaches a value close to 0.2. The deviations from Gaussian behavior observed for each type of hydrogens in PVE can be explained, as in the case of PI, in the frame of a simple model that considers a distribution of finite jumps underlying the motion of protons during the $\alpha$ process. The values found for the most probable jump distances are reasonable and for the main chain protons compare well with those deduced for PI main chain protons. This gives additional support to such simple scenario.

\section{ACKNOWLEDGMENTS}

We thank Dr. L. Willner for the sample preparation and Dr. W. Schweika for experimental support at the DNS instrument. This experiment was supported by the European Commission under the "Access to Research Infrastructures Action of the Human Potential Program" Contract No. HPRI2001-0268. J.C., A.A., A.N., and F.A. acknowledge the University of the Basque Country (Project 9/UPV00206.21513568/2001) and the Spanish Ministry of Science and Technology (Project MAT 2001/0070) for their support. A.N. acknowledges the FPI grant of the Spanish Ministery of Science and Technology. Support from "Donostia International Physics Center" is also acknowledged by A.A., J.C., F.A., and D.R.

${ }^{1}$ J. Colmenero, A. Alegría, A. Arbe, and B. Frick, Phys. Rev. Lett. 69, 478 (1992).

${ }^{2}$ A. Arbe, J. Colmenero, M. Monkenbusch, and D. Richter, Phys. Rev. Lett. 81, 590 (1998)

${ }^{3}$ J. Colmenero, F. Alvarez, and A. Arbe, Phys. Rev. E 65, 041804 (2002).

${ }^{4}$ A. Arbe, J. Colmenero, F. Alvarez, M. Monkenbusch, D. Richter, B. Farago, and B. Frick, Phys. Rev. E 67, 051802 (2003).

${ }^{5}$ W. Kob, C. Donati, S. J. Plimpton, P. H. Poole, and S. C. Glotzer, Phys. Rev. Lett. 79, 2827 (1997).

${ }^{6}$ F. Sciortino, P. Gallo, P. Tartaglia, and S.-H. Chen, Phys. Rev. E 54, 6331 (1996).

${ }^{7}$ S. Mossa, R. Di Leonardo, G. Ruocco, and M. Sampoli, Phys. Rev. E 62, $612(2000)$

${ }^{8}$ D. Caprion, J. Matsui, and H. R. Schober, Phys. Rev. Lett. 85, 4293 (2000).
${ }^{9}$ H. Sun, J. Comput. Chem. 15, 752 (1994); H. Sun, S. J. Mumby, J. R. Maple, and A. T. Hagler, J. Am. Chem. Soc. 116, 2978 (1994); H. Sun, Macromolecules 28, 701 (1995); 26, 5924 (1994); H. Sun, S. J. Mumby, J. R. Maple, and A. T. Hagler, J. Phys. Chem. 99, 5873 (1995).

${ }^{10}$ See, for example: H. Sun, J. Phys. Chem. B 102, 7338 (1998), http:// www.accelrys.com/doc

${ }^{11}$ D. N. Theodorou and U. W. Suter, Macromolecules 18, 1467 (1985).

${ }^{12}$ J. M. Carella, W. W. Grassley, and L. J. Fetters, Macromolecules 17, 2775 (1984).

${ }^{13}$ The structural relaxation time of our systems, i.e., the relaxation time of the density-density correlation function at the $Q$ value of the fist maximum of $S(Q)$, is $\tau_{s}=0.1 \mathrm{~ns}$ for $418 \mathrm{~K}$ [D. Richter, M. Monlenbusch, L. Willner, A. Arbe, J. Colmenero, and B. Farago, Europhys. Lett. 66, 239 (2004)]. Therefore, the equilibration time used for the first MD run (1 ns) is ten times $\tau_{s}$. For the last run of $2 \mathrm{~ns}$, the system was, in fact, equilibrated during the equilibration time plus the time of the previous MD runs, i.e., $2 \mathrm{~ns}$ in total (20 times $\tau_{s}$ ).

${ }^{14}$ J. C. Crocker and D. G. Grier, J. Colloid Interface Sci. 179, 298 (1996).

${ }^{15}$ A. H. Marcus, B. Lin, and S. A. Rice, Phys. Rev. E 53, 1765 (1996).

${ }^{16}$ S. W. Lovesey, Theory of Neutron Scattering from Condensed Matter (Clarendon, Oxford, 1984).

${ }^{17}$ G. L. Squires, Introduction to the Theory of Thermal Neutron Scattering (Dover, New York, 1996).

${ }^{18}$ M. Bée, Quasielastic Neutron Scattering (Adam Hilger, Bristol, 1988).

${ }^{19}$ T. Springer, Quasielastic Neutron Scattering for the Investigation of Diffusive Motions in Solids, Liquids, Springer Tracts in Modern Physics Vol. 64 (Springer, Berlin, 1972).

${ }^{20}$ R. Zorn, Phys. Rev. B 55, 6249 (1997).

${ }^{21}$ A. Rahman, K. S. Singwi, and A. Sjölander, Phys. Rev. 126, 986 (1962).

${ }^{22}$ M. Morton and L. J. Fetters, Rubber Chem. Technol. 48, 359 (1975).

${ }^{23}$ R. Zorn, G. B. McKenna, L. Willner, and D. Richter, Macromolecules 28, 8552 (1995).

${ }^{24}$ F. Mezei, Neutron Spin Echo, Lecture Notes in Physics Vol. 28 (SpringerVerlag, Heidelberg, 1980).

${ }^{25}$ B. Farago, Physica B 241-241, 113 (1997).

${ }^{26}$ Neutron Spin Echo Spectroscopy: Basics, Trends and Applications, edited by F. Mezei, C. Pappas, and T. Gutberlet (Springer, Berlin, 2003).

${ }^{27}$ P. G. de Gennes, Physica (Amsterdam) 25, 825 (1959).

${ }^{28}$ D. Richter, B. Frick, and B. Farago, Phys. Rev. Lett. 61, 2465 (1988).

${ }^{29}$ B. Farago, A. Arbe, J. Colmenero, R. Faust, U. Buchenan, and D. Richter, Phys. Rev. E 65, 051803 (2002).

${ }^{30}$ K. Binder, J. Baschnagel, and W. Paul, Prog. Polym. Sci. 28, 115 (2003).

${ }^{31}$ A. Van Zon and S. W. De Leeuw, Phys. Rev. E 60, 6942 (1999).

${ }^{32}$ J. Colmenero, A. Arbe, A. Alegría, and K. L. Ngai, J. Non-Cryst. Solids 172-174, 229 (1994).

${ }^{33}$ A. Chahid, A. Alegría, and J. Colmenero, Macromolecules 27, 3282 (1994).

${ }^{34}$ R. Zorn, B. Frick, and L. J. Fetters, J. Chem. Phys. 116, 845 (2002).

${ }^{35}$ A. Arbe, J. Colmenero, F. Alvarez, M. Monkenbusch, D. Richter, B. Farago, and B. Frick, Phys. Rev. Lett. 89, 245701 (2002).

${ }^{36}$ J. Colmenero, F. Alvarez, A. Narros, A. Arbe, M. Monkenbusch, D. Richter, B. Farago; Proceedings of the Conference: Slow Dynamics in Complex Systems, Sendai, November 2004

${ }^{37}$ W. K. Kegel and A. Van Blaaderen, Science 287, 290 (2000); E. R. Weeks, J. C. Crocker, A. C. Levitt, A. Schofield, and D. A. Weitz, ibid. 287, 627 (2000).

${ }^{38}$ W. Götze, in Liquids, Freezing, Glass Transition, edited by J. P. Hansen, D. Levesque, J. Zinn-Justin (North-Holland, Amsterdam, 1991), p. 287.

${ }^{39}$ An Introduction to the Liquid State, edited by P. A. Egelstaff (Oxford University Press, New York, 1992).

${ }^{40}$ K. S. Singwi, A. Sjölander, Phys. Rev. 119, 863 (1960).

${ }^{41}$ J. Colmenero, A. Arbe, F. Alvarez, M. Monkenbusch, D. Richter, B. Farago, and B. Frick, J. Phys.: Condens. Matter 15, S1127 (2003).

${ }^{42}$ A. Narros, J. Colmenero, A. Arbe, F. Alvarez, M. Monkenbusch, and D. Richter (unpublished). 